\title{
Análise da hidratação de grãos de alpiste
}

\author{
Jemima F. Lisbôa ${ }^{1}$, José N. Silva ${ }^{2}$, Mônica T. Cavalcanti ${ }^{3}$, Emanuelle M. C. A. Silva ${ }^{4}$ \& Mônica C. Gonçalves ${ }^{5}$
}

\section{Palavras-chave:}

difusão

Peleg

Phalaris canariensis L.

\begin{abstract}
R E S U M O
Neste trabalho se objetivou estudar o fenômeno de hidratação em grãos de alpiste (Phalaris canariensis L.) em diferentes temperaturas. Foram utilizados grãos secos de alpiste, embebidos em água destilada, nas temperaturas de 10,25 e $45^{\circ} \mathrm{C}$. O aumento da temperatura resultou em aumento da taxa de absorção de água e o modelo de Peleg se ajustou satisfatoriamente aos dados experimentais. $\mathrm{O}$ coeficiente efetivo de difusão aumentou com a variação crescente da temperatura, de 2,396 até $2,428 \times 10^{-13} \mathrm{~m}^{2} \mathrm{~s}^{-1}$. A dependência do coeficiente efetivo de difusão com a temperatura pode ser descrita pela relação de Arrhenius apresentando, para a faixa de temperatura estudada, a magnitude da energia de ativação aparente para o processo de 39,239 $\pm 4,3463$.
\end{abstract}

Key words:

diffusion

Peleg

Phalaris canariensis L.

\section{Analysis of hydration of grains of birdseed}

\section{A B S T R A C T}

The objective of this work was to study the hydration of grains of birdseed (Phalaris canariensis L.) at different temperatures. Dried birdseed beans were used embedded in distilled water at temperatures of 10,25 and $45^{\circ} \mathrm{C}$. Increasing the temperature resulted in higher water absorption rate and the model of Peleg adjusted satisfactorily to experimental data. The effective diffusion coefficient increased with increasing temperature from 2.396 to $2.428 \times 10^{-13} \mathrm{~m}^{2} \mathrm{~s}^{-1}$. The dependence of the effective diffusion coefficient on temperature can be described by the Arrhenius relationship, presenting, for the studied temperature range, the magnitude of the apparent activation energy for the process of $39.239 \pm 4.3463$.

Protocolo 039-2014 - 04/02/2014 • Aprovado em 31/10/2014 • Publicado em 26/01/2015

${ }^{1}$ PPGEA/CTRN/UFCG, Campina Grande, Paraíba, E-mail: jemimaufcg_@hotmail.com

${ }^{2}$ UAEQ/CCT/UFCG, Campina Grande, Paraíba, E-mail: nilton@deq.ufcg.edu.br

${ }^{3}$ UATA/CCTA/UFCG, Pombal, Paraíba, E-mail: monicatejoc@yahoo.com.br (Autora correspondente)

${ }^{4}$ UAEA/CTRN/UFCG, Campina Grande, Paraíba, E-mail: emanuellecavelino@gmail.com

${ }^{5}$ UATA/CCTA/UFCG, Pombal, Paraíba, E-mail: monica.goncalves@ccta.ufcg.edu.br 


\section{INTRODUÇÃo}

O alpiste, Phalaris canariensis L. (Graminaceae), é um tipo de grão natural da região do Mediterrâneo, muito utilizado para a alimentação de pássaros quanto na alimentação humana, em sopas e doces e também na produção de cola, em indústrias têxteis (Reitz, 1982).

O processo de hidratação de grãos está presente na caracterização da qualidade fisiológica do grão, na extração de algum constituinte de interesse, no cozimento, na redução ou eliminação de fatores antinutricionais existentes nos grãos e na melhora da digestibilidade (Omoto et al., 2009). A umidificação depende de variáveis, como a temperatura e o teor de água inicial do grão. Dados de variação do teor de água de grãos em função do tempo podem ser empregados para descrever o comportamento dos dados de hidratação e interpretá-los por intermédio de dois tipos básicos de modelos: empíricos e fenomenológicos.

A hidratação tem sido entendida como um processo físico diretamente relacionado com as características de permeabilidade do envoltório (pericarpo) e as propriedades dos coloides constituintes dos grãos (Woodstock,1988; Bewley \& Black, 1994). A cinética de hidratação tem sido amplamente caracterizada por produtos como grãos de cereais e leguminosas (Abu-Ghannam \& Emckenna, 1997; Lucas et al., 2007).

Estudos envolvendo a cinética de adsorção e absorção em diversas variedades de grãos, tais como soja (Fracasso et al., 2014), feijão (Resende et al., 2010), arroz parbolizado (Botelho et al., 2010) e cereais (Lucas et al., 2007), entre outros produtos industrializados, têm demonstrado o interesse e sua importância. Nos vários trabalhos citados na literatura tem-se avaliado a influência da temperatura na taxa e na quantidade de hidratação dos grãos.

A modelagem matemática permite predizer e simular o comportamento de determinado parâmetro e processos, podendo ser utilizado modelos empíricos e fenomenológicos. Os modelos empíricos, apesar de normalmente não possuírem uma fundamentação teórica são, em geral, mais simples e de fácil aplicação visto que estão fundamentados na análise de dados experimentais, na análise dimensional e na análise estatística; por sua vez, os modelos fenomenológicos baseados em teorias e leis, são mais complexos e envolvem parâmetros que refletem, com boa aproximação, o sentido físico do sistema podendo esses parâmetros também serem obtidos no intuito de representar o sistema real.

O modelo de difusão líquida é o mais comum entre os modelos teóricos aplicados ao processo de hidratação. Tal modelo é aplicado em processos de secagem e de hidratação de sólidos considerando-se, como mecanismo principal, a difusão líquida baseada na Lei de Fick, que estabelece uma relação entre as taxas de variação temporal e espacial da concentração de água, por meio do coeficiente de difusão. A difusão de água em grãos ocorre devido à existência de uma força motriz, ou seja, um gradiente de concentração de água entre a superfície e o interior do produto. No entanto, alterações físico-químicas ocorrem em paralelo com a difusão de água nos grãos sendo observadas alterações na composição química e na estrutura física. Como os grãos podem, em sua maioria, ser capilares porosos os possíveis mecanismos de transporte de água são: difusão líquida, difusão capilar, difusão na superfície, fluxo hidrodinâmico, difusão de vapor e difusão térmica (Brooker et al., 1992).

Informações sobre o mecanismo de transporte de água podem ser obtidas a partir das isotermas de hidratação obtidas experimentalmente e possibilitando a determinação do coeficiente efetivo de difusão. Segundo Roca et al. (2008), o coeficiente de difusão determinado experimentalmente é considerado como efetivo ou aparente haja vista que os modelos baseados na segunda Lei de Fick não são rigorosamente representativos dos diversos mecanismos que prevalecem no transporte de água em grãos.

Muitos pesquisadores têm verificado que a dependência do coeficiente efetivo de difusão com a temperatura pode ser expressa por meio da representação de Arrhenius (Lucas et al., 2007; Botelho et al., 2010; Resende et al., 2010). Dos vários modelos empíricos propostos, o que vem sendo frequentemente utilizado para predizer a hidratação de diversos tipos de grãos é o proposto por Peleg (1988). A principal vantagem de tal modelo é a simplicidade com relação aos demais modelos teóricos e empíricos. Este modelo já foi utilizado para descrever a cinética de hidratação em soja (Fracasso et al., 2014), para o feijão variedade Adzuki (Resende et al., 2009), dentre outros.

A partir do exposto tem-se, por objetivo, modelar, para diferentes temperaturas, a cinética de hidratação dos grãos de alpiste e verificar a influência deste processo na difusão de água para o interior do grão.

\section{Material e Métodos}

Este trabalho foi realizado no Laboratório de Grãos e Cereais pertencentes à Unidade Acadêmica de Tecnologia de Alimentos, do Centro de Ciências Agroalimentar localizado no campus da Universidade Federal de Campina Grande, UFCG, Pombal, PB.

Utilizaram-se, para a realização dos experimentos, grãos de alpiste beneficiados industrialmente e adquiridos em comércio local do município de Pombal, $\mathrm{PB}$, onde foi feita, inicialmente, a seleção do material a fim de eliminar grãos defeituosos e materiais estranhos.

Antes do processo de hidratação foram utilizadas amostras de $100 \mathrm{~g}$ de grãos de alpiste, previamente secadas em estufa possibilitando obter o teor de água próximo de zero; em seguida, os grãos foram embebidos com água destilada numa razão 4/1 volumes de água/grão e submetidos às temperaturas de 10,25 e $40{ }^{\circ} \mathrm{C}$, em banho-maria, durante o período de treze horas, em triplicata; durante este período as amostras foram pesadas, periodicamente, em uma balança digital com resolução de $0,0001 \mathrm{~g}$, na qual os grãos foram retirados da imersão e deixados sobre papel toalha por três minutos, para que a água superficial fosse retirada, pesadas e posteriormente retornavam à imersão. O teor de água foi determinado tomando-se como base o aumento de massa total das amostras em relação à massa seca inicial.

Para a modelagem da hidratação do alpiste utilizou-se o tradicional modelo de Peleg (1988), Eq.1, tendo tal modelo uma das grandes vantagens, ou seja, o ganho de tempo para predizer a cinética de sorção de água em alimentos possibilitando o cálculo do teor de água de equilíbrio. 


$$
X(t)=X_{0}(t)+\frac{t}{K_{1}+K_{2} t}
$$

em que as constantes $\mathrm{K}_{1}$ e $\mathrm{K}_{2}$ do modelo proposto para modelagem do presente processo foram obtidas pelo ajuste da Equação de Peleg linearizada, conforme descrito na Eq. 2:

$$
\frac{1}{X(t)-X_{0}(t)}=K_{1} \frac{1}{t}+K_{2}
$$

ou, ainda,

$$
\mathrm{X}_{(\mathrm{t})}^{* \mathrm{exp}}=\mathrm{K}_{1} \mathrm{t}^{*}+\mathrm{K}_{2}
$$

onde:

$\mathrm{X}(\mathrm{t})$ - teor de água para determinado tempo, $\mathrm{kg}_{\mathrm{a}} 100 \mathrm{~kg}_{\mathrm{ms}}{ }^{-1}$ $\mathrm{X}_{0}(\mathrm{t})$ - teor de água inicial do produto, $\mathrm{g}_{\mathrm{a}} 100 \mathrm{~kg}_{\mathrm{ms}}{ }^{-1}$

$\mathrm{K}_{1} \quad$ - taxa constante de Peleg, $100 \mathrm{~h} \mathrm{~kg}_{\mathrm{ms}} \mathrm{kg}_{\mathrm{a}}{ }^{-1}$

$\mathrm{K}_{2}$ - capacidade constante de Peleg, $100 \mathrm{~kg}_{\mathrm{ms}} \mathrm{kg}_{\mathrm{a}}{ }^{-1}$

$\mathrm{t}$ - tempo, h

Sendo também $\mathrm{X}^{\star \exp }(\mathrm{t})=1 /(\mathrm{X}(\mathrm{t})-\mathrm{X} 0(\mathrm{t}))$ e $\mathrm{t}^{*}=1 / \mathrm{t}$.

$\mathrm{O}$ teor de água de equilíbrio pode ser obtido pela própria Eq. 1, quando o tempo tende ao infinito, resultando na Eq. 4.

$$
\mathrm{X}_{\mathrm{eq}}=\mathrm{X}_{0}(\mathrm{t})+\frac{1}{\mathrm{~K}_{2}}
$$

em que:

$$
\mathrm{X}_{\mathrm{eq}} \text { - teor de água de equilíbrio, } \mathrm{kg}_{\mathrm{a}} 100 \mathrm{~kg}_{\mathrm{ms}}{ }^{-1}
$$

O coeficiente efetivo de difusão foi obtido por meio do ajuste do modelo matemático da difusão líquida, descrito pela Eq.4, aos dados experimentais de hidratação do alpiste; esta equação é a solução analítica de Crank (1975) para uma geometria esférica desconsiderando-se a expansão volumétrica dos grãos porém considerando a condição de contorno de equilíbrio de umidade conhecida na superfície do grão.

$$
\mathrm{R}_{\mathrm{U}}=\frac{\mathrm{X}(\mathrm{t})-\mathrm{X}_{\mathrm{eq}}}{\mathrm{X}_{0}(\mathrm{t})-\mathrm{X}_{\mathrm{eq}}}=\frac{6}{\pi^{2}} \sum_{\mathrm{n}=1}^{\infty} \frac{1}{\lambda_{\mathrm{n}}^{2}} \mathrm{e}^{\left[\frac{-\lambda_{\mathrm{n}}^{2} \pi^{2} \mathrm{D}_{\mathrm{eft}}}{9}\left(\frac{3}{\mathrm{r}}\right)^{2}\right]}
$$

sendo que:

$\mathrm{R}_{\mathrm{u}}$ - razão de umidade

$\lambda_{\mathrm{n}} \quad$ - raízes da função de Bessel de ordem zero

$\mathrm{D}_{\text {ef }}$ - coeficiente efetivo de difusão, $\mathrm{m}^{2} \mathrm{~s}^{-1}$

$r \quad$ - raio equivalente, $m$

A solução analítica desta equação se apresenta na forma de uma série infinita de rápida convergência; desta forma, um número finito de termos (n) pode ser utilizado para uma precisão especificada.

$\mathrm{O}$ raio equivalente de um grão pode ser definido como o raio da esfera com mesmo volume. Para se determinar o volume médio dos grãos de alpiste considerou-se o alpiste como sendo um elipsoide; para o cálculo do seu volume tomou-se uma amostra de trinta e dois grãos, na qual foram realizadas medidas no comprimento, largura e espessura, com a ajuda de um paquímetro digital com resolução de $0,001 \mathrm{~mm}$ sendo que foram consideradas, como diâmetro do cilindro, as médias das medidas da largura e espessura. $\mathrm{O}$ raio equivalente encontrado neste trabalho foi de $0,00265 \mathrm{~m}$.

A dependência do coeficiente efetivo de difusão com a temperatura foi verificada utilizando-se a relação de Arrhenius, Eq. 6:

$$
\mathrm{D}_{\text {ef }}=\mathrm{D}_{0} \mathrm{e}^{\left[\frac{-\mathrm{E}_{\mathrm{a}}}{\mathrm{RT}_{\mathrm{a}}}\right]}
$$

onde:

$\mathrm{E}_{\mathrm{a}}$ - energia de ativação, $\mathrm{kJ} \mathrm{mol}^{-1}$

$\mathrm{R}^{\mathrm{a}} \quad$ - constante universal dos gases, $\mathrm{kJ} \mathrm{mol}^{-1} \mathrm{~K}^{-1}$

$\mathrm{T}_{\mathrm{a}}$ - temperatura absoluta, $\mathrm{K}$

$\mathrm{D}_{0}$ - fator pré-exponencial equivalente ao coeficiente de difusão para temperaturas tendendo ao infinito, $\mathrm{m}^{2} \mathrm{~s}^{-2}$

O coeficiente efetivo de difusão foi obtido por meio de regressão não linear da Eq. 4, com aproximação de sete termos pelo método Gradiente Reduzido Generalizado Gauss, utilizando-se a ferramenta Solver ${ }^{\circ}$ do Excel $^{\circ}$.

As equações para a análise estatística, apresentadas a seguir, foram obtidas a partir do método dos mínimos quadrados utilizando-se o modelo da Eq. 2 juntamente com fundamentos de estatísticas e aplicação do operador esperança, de acordo com Schwaab \& Pinto (2007).

Para verificar o grau de ajuste do modelo foram consideradas as magnitudes do coeficiente de correlação do modelo $\left(\rho^{\mathrm{m}}\right)$, Eq. 7, as variâncias e covariâncias dos parâmetros dadas por $\sigma_{\mathrm{K} 1}^{2}, \sigma_{\mathrm{K} 2}^{2} \mathrm{e} \sigma_{\mathrm{K} 1 \mathrm{~K} 2}^{2}$, respectivamente, apresentadas nas Eqs. 8 a 10.

$$
\rho^{m}=\frac{\sum_{i=1}^{n}\left(X_{i}^{\exp }(t)-\bar{X}_{i}^{\exp }\right)\left(X_{i}^{m}(t)-\bar{X}_{i}^{m}\right)}{\sqrt{\sum_{i=1}^{n}\left(X_{i}^{m}(t)-\bar{X}_{i}^{\exp }\right)^{2} \sum_{i=1}^{n}\left(X_{i}^{m}(t)-\bar{X}_{i}^{m}\right)^{2}}}
$$

$$
\sigma_{\mathrm{K}_{1}}^{2}=\sigma_{\mathrm{X}^{* \times \mathrm{xp}}}^{2} \frac{\mathrm{n}}{\mathrm{n} \sum_{\mathrm{i}=1}^{\mathrm{n}}(\mathrm{t} *)^{2}-\left[\sum_{\mathrm{i}=1}^{\mathrm{n}}(\mathrm{t} *)\right]^{2}}
$$

$$
\begin{gathered}
\sigma_{\mathrm{K}_{2}}^{2}=\sigma_{\mathrm{X}^{* \text { exp }}}^{2} \frac{\sum_{\mathrm{i}=1}^{\mathrm{n}}(\mathrm{t} *)^{2}}{\mathrm{n} \sum_{\mathrm{i}=1}^{\mathrm{n}}(\mathrm{t} *)^{2}-\left[\sum_{\mathrm{i}=1}^{\mathrm{n}}(\mathrm{t} *)\right]^{2}} \\
\sigma_{\mathrm{K}_{1} \mathrm{~K}_{2}}^{2}=\sigma_{\mathrm{X}^{* \times p p}}^{2} \frac{-\sum_{\mathrm{i}=1}^{\mathrm{n}}(\mathrm{t} *)}{\mathrm{n} \sum_{\mathrm{i}=1}^{\mathrm{n}}(\mathrm{t} *)^{2}-\left[\sum_{\mathrm{i}=1}^{\mathrm{n}}(\mathrm{t} *)\right]^{2}}
\end{gathered}
$$

sendo:

$\mathrm{X}_{\mathrm{i}}^{\exp }(\mathrm{t})$ - valor experimental

$\mathrm{X}_{\mathrm{i}}^{\mathrm{t} \text { teor }}(\mathrm{t})$ - valor estimado pelo modelo 
n - número de dados observados

$t^{*} \quad$ - inverso do tempo $(1 / t)$ de amostragem

O coeficiente de correlação paramétrica $\left(\rho_{\mathrm{K} 1 \mathrm{~K} 2}\right)$ foi obtido a partir da Eq. 11 no intuito de avaliar o grau de relação entre as mesmas. Os erros de predição foram determinados pela Eq. 12; o teste $\mathrm{F}$ foi aplicado visando avaliar se as variâncias de predição e experimental foram semelhantes estatisticamente permitindo verificar se o modelo poderia ser considerado satisfatório.

$$
\begin{gathered}
\rho_{\alpha \beta}=\frac{\sigma_{\alpha \beta}^{2}}{\sigma_{\alpha} \sigma_{\beta}} \\
\hat{\sigma} \frac{2}{y}=\sigma \frac{2}{y} \frac{n x^{2}-2 x \sum_{i=1}^{n} x_{i}^{e}+\sum_{i=1}^{n}\left(x_{i}^{e}\right)^{2}}{n \sum_{i=1}^{n}\left(x_{i}^{e}\right)^{2}-\left[\sum_{i=1}^{n}\left(x_{i}^{e}\right)\right]^{2}}
\end{gathered}
$$

\section{Resultados e Discussão}

Na Figura 1 estão expostas as variações do teor de água do alpiste em função do tempo nas temperaturas avaliadas.

Percebe-se que o alpiste exibiu um comportamento característico de diversos grãos submetidos ao processo de hidratação. Observa-se, no início do processo, que a hidratação exibe alta taxa de absorção de água tendendo a diminuir com o tempo, à medida que o teor de água do produto se aproxima do equilíbrio.

O comportamento observado na Figura 1, similar ao justificado por Bello et al. (2007), pode ser atribuído à inibição capilar das camadas externas do pericarpo que apressaria a absorção de água. Semelhante à explicação dada por Bello et al. (2007), a estrutura matricial dos vários tecidos do grão de alpiste também poderia contribuir para as altas taxas de absorção de água.

O tempo total de $13 \mathrm{~h}$ para cada experimento foi suficiente para chegar próximo ao equilíbrio mas é possível observar um aumento gradativo na absorção de água com o aumento de temperatura. Como não foi alcançado o patamar de equilíbrio para o intervalo estudado, possivelmente não se tenha atingido a temperatura de gelatinização do amido para este grão uma vez que não foi verificado comportamento abrupto na absorção de água.

Para verificar a qualidade do ajuste do modelo de Peleg para representar a cinética da hidratação dos grãos de alpiste, o modelo foi ajustado aos dados experimentais

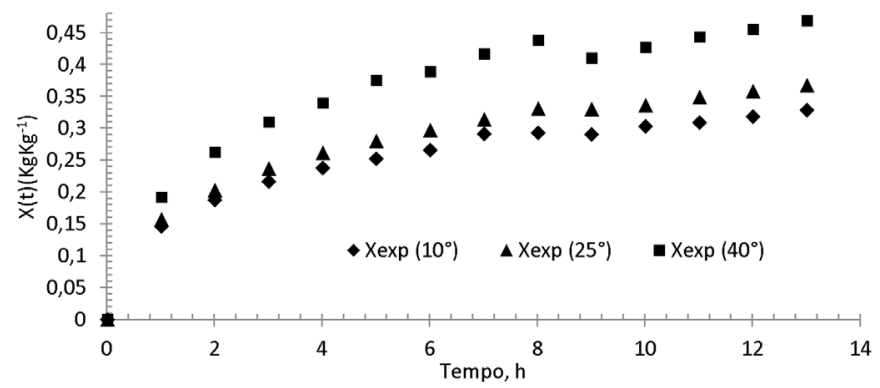

Figura 1. Valores experimentais para o teor de água no grão de alpiste para as temperaturas de 10,25 e $40{ }^{\circ} \mathrm{C}$ sendo determinados seus coeficientes para cada temperatura estudada. A determinação dos parâmetros cinéticos foi realizada a partir da minimização da função da soma dos quadrados entre os dados experimentais e a função do modelo linearizado de Peleg da Eq. 2, resultando nos estimadores, de acordo com as equações seguintes:

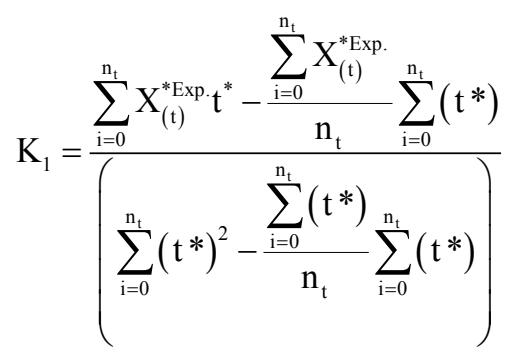

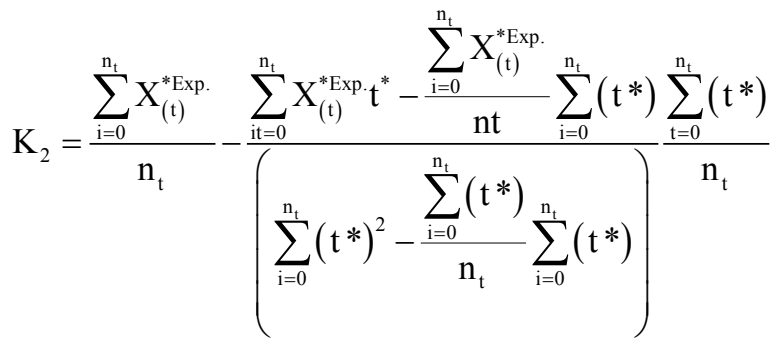

Verifica-se, na Figura 2, um ajuste aceitável do modelo aos dados experimentais, o que pode ser comprovado pelos valores obtidos do teste $p$-value menores que o valor do nível de significancia $a$ adotado de $5 \%$, e a correspondência entre os valores observados e estimados pelo modelo para todas as temperaturas de hidratação.

$\mathrm{Na}$ Tabela 1 estão apresentados os valores dos coeficientes $\mathrm{K}_{1}$ e $\mathrm{K}_{2}$ do modelo, assim como os intervalos de confiança para os mesmos e do coeficiente de correlação $\left(\rho^{\mathrm{m}}\right)$; nesta mesma tabela estão apresentados os teores de água de equilíbrio $\left(\mathrm{K}_{\mathrm{eq}}\right)$ obtidos pela Eq. 3 .

De acordo com Turhan et al. (2002), a constante $K_{1}$ do modelo de Peleg está relacionada com a taxa de transferência de massa sendo que, quanto menor os valores de $\mathrm{K}_{1}$, maiores

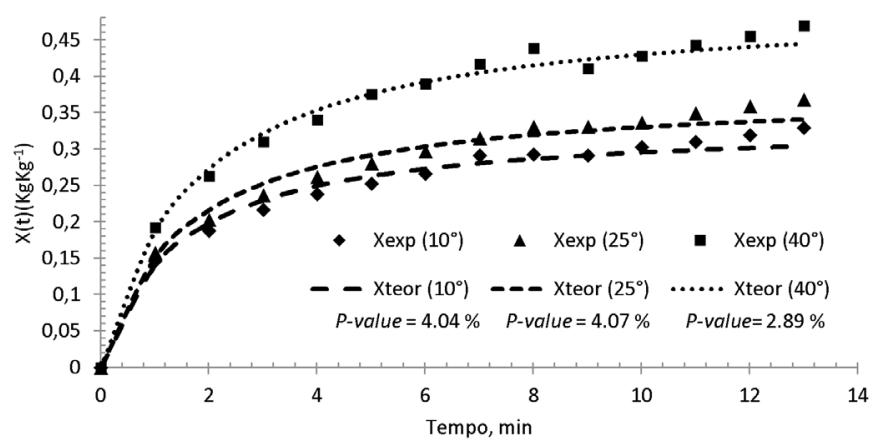

Figura 2. Valores observados e estimados pelo modelo de

\begin{tabular}{|c|c|c|c|c|}
\hline $\begin{array}{l}\mathrm{T} \\
\left({ }^{\circ} \mathrm{C}\right)\end{array}$ & $\underset{\left(\operatorname{minkg} \mathrm{ms}_{\mathrm{ms}} \mathrm{kg}_{\mathrm{a}}^{-1}\right)}{\mathrm{K}_{1}}$ & $\begin{array}{c}K_{2} \\
\left(\mathrm{~kg}_{\mathrm{ms}} \mathrm{kg}_{\mathrm{a}}{ }^{-1}\right)\end{array}$ & $\begin{array}{c}X_{\mathrm{eq}} \\
\left(\mathrm{kg}_{\mathrm{a}} \mathrm{kg}_{\mathrm{mm}}^{-1}\right)\end{array}$ & $\rho^{m}$ \\
\hline 10 & $248,379 \pm 2,174$ & $2,9623 \pm 0,012595$ & 0,3376 & 0,991779 \\
\hline 25 & $239,073 \pm 2,182$ & $2,6239 \pm 0,012639$ & 0,3811 & 0,995128 \\
\hline 40 & $200,146 \pm 2,390$ & $1,9868 \pm 0,012639$ & 0,5033 & 0,995202 \\
\hline
\end{tabular}
Peleg para as temperaturas de 10,25 e $40^{\circ} \mathrm{C}$

Tabela1. Parâmetros, umidade de equilíbrio em função da temperatura de hidratação 
serão as taxas iniciais de absorção de água pelo grão. Segundo Cunningham et al. (2007) e Maskan (2002), este coeficiente pode, ainda, ser relacionado com o coeficiente de difusão. Com avaliação do comportamento de tais coeficientes em função da temperatura de hidratação verificou-se que esses parâmetros decresceram com o aumento da temperatura segundo uma relação quadrática, como mostrado a Tabela 2 .

Tabela 2. Funções dos parâmetros do modelo em função da temperatura

\begin{tabular}{ccc}
\hline Parâmetro & Equação & $\mathbf{R}^{\mathbf{2}}$ \\
$\mathrm{K}_{1}$ & $-0,0658 \mathrm{~T}^{2}+1,6835 \mathrm{~T}+238,13$ & 0,9998 \\
$\mathrm{~K}_{2}$ & $-0,0007 \mathrm{~T}^{2}+0,0007 \mathrm{~T}+3,0221$ & 0,9995 \\
\hline
\end{tabular}

O comportamento dos coeficientes para esse experimento era previsto, de vez que resultados semelhantes foram observados por vários autores (Resende \& Corrêa, 2007; Cunningham et al., 2007; Resende et al., 2010; Fracasso et al., 2014).

Considerando que as constantes $\mathrm{K}_{1} \mathrm{e} \mathrm{K}_{2}$ podem ser expressas em função da temperatura por meio das equações da Tabela 2 , o modelo de Peleg pode ser descrito de acordo com a Eq. 10.

$$
\begin{aligned}
X(t) & =X_{0}(t)+\left\{t /\left[\left(-0,658 T^{2}+1,6835 T+238,3\right)+\right.\right. \\
& \left.\left.+\left(-0,0007 T^{2}+0,0007 T+3,0221\right) t\right]\right\}
\end{aligned}
$$

onde:

$$
\mathrm{T} \text { - temperatura, }{ }^{\circ} \mathrm{C}
$$

Na Figura 3 se apresenta o gráfico de correspondência entre os valores observados experimentais e os estimados pelo modelo de Peleg, com seus respectivos coeficientes nas temperaturas estudadas para 10,25 e $40^{\circ} \mathrm{C}$, tal como o intervalo de confiança com nível de significância a de 5\%.

Pela correspondência entre os valores experimentais e os estimados e pela magnitude do valor do coeficiente de correlação $\rho^{m}$, nota-se que, com seus coeficientes em função da temperatura, o modelo de Peleg descreve adequadamente o processo de hidratação do alpiste.

Os valores do coeficiente efetivo de difusão para cada condição de hidratação do alpiste estão apresentados na Tabela 3.

Com base nos resultados da Tabela 3, observa-se que os valores do coeficiente efetivo de difusão aumentaram com a elevação da temperatura variando de $2,396 \times 10^{-13}$ a

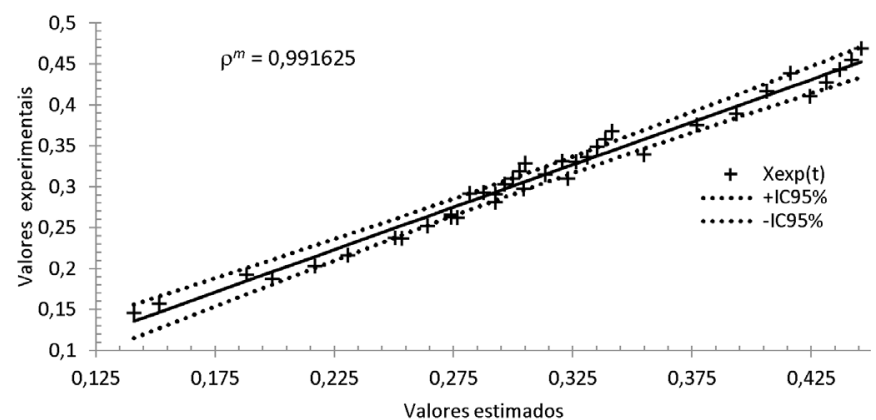

Figura 3. Correspondência entre os valores experimentais e os estimados pelo modelo de Peleg com seus coeficientes variando em função da temperatura de hidratação
Tabela 3. Coeficiente efetivo de difusão para o alpiste em função da temperatura de hidratação

\begin{tabular}{ccc}
\hline Temperatura $\left({ }^{\circ} \mathbf{C}\right)$ & Coeficiente de difusão efetiva $\mathbf{x} \mathbf{1 0}^{\mathbf{1 3}}\left(\mathbf{m}^{\mathbf{2}} \mathbf{s}^{-1}\right)$ \\
10 & 2,396 & $\pm 0,03498$ \\
25 & 2,414 & $\pm 0,03456$ \\
40 & 2,428 & $\pm 0,03437$ \\
\hline
\end{tabular}

$2,428 \times 10^{-13} \mathrm{~m}^{2} \mathrm{~s}^{-1}$, com o desvio de $0,034 \times 10^{-13} \mathrm{~m}^{2} \mathrm{~s}^{-1}$, com nível de significância $\alpha=5 \%$. Com o aumento da temperatura a viscosidade da água diminui e, sendo esta propriedade uma medida da resistência do fluído ao escoamento, suas variações implicam em alterações na difusão da água nos capilares do grão, de modo a favorecer sua movimentação no produto. Outro fator ao qual pode ser atribuída esta variação do coeficiente efetivo de difusão é que, com o aumento da temperatura, aumenta o nível de vibração das moléculas da água contribuindo para uma difusão mais rápida.

Vários autores relatam o comportamento do coeficiente efetivo de difusão. Botelho et al. (2010) obtiveram, estudando a hidratação do arroz na parboilização, valores de 0,8 a 9,18 x $10^{-11}\left(\mathrm{~m}^{2} \mathrm{~s}^{-1}\right)$, Siqueira et al. (2012) relataram, estudando grãos e frutos de pinhão-manso, valores entre $9,29 \times 10^{-10}$ a $41,48 \mathrm{x}$ $10^{-10} \mathrm{~m}^{2} \mathrm{~s}^{-1}$.

A dependência do coeficiente efetivo de difusão com a temperatura, expressa por meio da relação de Arrhenius, está apresentada na Figura 4 com a respectiva Equação de Arrhenius linearizada.

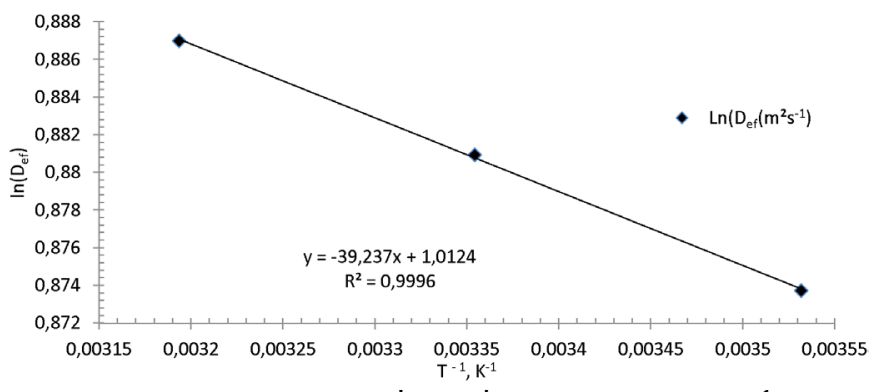

Figura 4. Representação de Arrhenius para o coeficiente efetivo de difusão para os grãos de alpiste durante o processo de hidratação

Nota-se, para o intervalo de temperatura de 10 a $40{ }^{\circ} \mathrm{C}$, que existe uma uniformidade de variação do coeficiente efetivo de difusão para esta faixa de temperatura.

Assim, para determinação da energia de ativação da difusão de água no alpiste durante o processo de hidratação e considerando a faixa de temperatura estudada, a Eq. 11 é o resultado da Equação de Arrhenius linearizada.

$$
\operatorname{Ln}(\text { Def })=1,0125-39,237 \frac{1}{T}
$$

Verifica-se, pela Eq. 11, que a magnitude do valor da razão da energia de ativação $\left(E_{a}\right)$ pela constante universal $(R)$ foi de 39,237. Tal valor permite mostrar que a hidratação de grãos de alpiste apresenta nível cinético de hidratação próximo aos obtidos por Kashaninejad et al. (2007) para Nozes de pistachio, 30,79, também de acordo com Resende et al. (2010) para arroz em casca, 34,80 e para o feijão-verde, 39,47 (Senadeera et al., 2003). 


\section{Conclusões}

1. A hidratação do alpiste pode ser descrita satisfatoriamente pelo modelo de Peleg.

2. As constantes $K_{1}$ e $K_{2}$ do modelo de Peleg decrescem com o aumento da temperatura de hidratação.

3. A taxa de hidratação aumenta com a elevação da temperatura com o coeficiente efetivo de difusão variando de 2,396 a 2,428 $\times$ $10^{-13} \mathrm{~m}^{2} \mathrm{~s}^{-1}$ para a faixa de temperatura de 10 a $40{ }^{\circ} \mathrm{C}$.

4. A magnitude da energia de ativação aparente foi de 39,239 .

\section{Literatura Citada}

Abu-Ghannam, N.; Emckenna, B. Hydration kinetics of red kidney beans. Journal of Food Science, v.62, p.520-523, 1997. http:// dx.doi.org/10.1111/j.1365-2621.1997.tb04420.x

Bello, M.; Tolaba, M. P.; Suarez, C. Water absorption and starch gelatinization in whole rice grain during soaking. Lebensmittel Wissenschaft and Technologie, v.40, p.313-318, 2007.

Bewley, J. D.; Black, M. Seeds: Physiology of development and germination. 2.ed. New York: Plenum Press, 1994. 445p. http:// dx.doi.org/10.1007/978-1-4899-1002-8

Botelho, F. M.; Corrêa, P. C.; Goneli, A. L. D.; Martins, M. A.; Baptestini, F. M. Análise da hidratação do arroz na parboilização. Ciência e Tecnologia de Alimentos, v.30, p.71-718, 2010. http:// dx.doi.org/10.1590/S0101-20612010000300023

Brooker, D. B.; Bakker-Arkema, F. W.; Hall, C. W. Drying and storage of grains and oilseeds.Westport: The AVI Publishing Company, 1992. 450p.

Crank, J. The mathematics of diffusion. Oxford University Press, 2 ed. London, 1975. 424p.

Cunningham, S. E.; Mcminn, W. A. M.; Richardson, P. S. Modelling water absorption of pasta during soaking. Journal of Food Engineering, v.82, p.600-607, 2007. http://dx.doi.org/10.1016/j. jfoodeng.2007.03.018

Fracasso, A. F. ; Perussello, C. A. ; Haminiuk, C. W. I. ; Jorge, L. M. M.; Jorge, R. M. M. Hydration kinetics of soybeans: transgenic and conventional cultivars. Journal of Cereal Science, v.60, p.584588,2014

Kashaninejad, M.; Mortazavi, A.; Safekordi, A.; Tabil, L. G. Thinlayer drying characteristics and modeling of pistachio nuts. Journal of Food Engineering, v.78, p.98-108, 2007. http://dx.doi. org/10.1016/j.jfoodeng.2005.09.007

Lucas, T.; Ray, D. L.; Mariette, F. Kinetics of water absorption and solute leaching during soaking of breakfast cereals. Journal of Food Engineering, v.80, p.377-384, 2007. http://dx.doi. org/10.1016/j.jfoodeng.2005.11.006
Maskan, M. Effect of processing on hydration kinetics of three wheat products of the same variety. Journal of Food Engineering, v.52, p.337-341, 2002. http://dx.doi.org/10.1016/S02608774(01)00124-8

Omoto, E. S.; Andrade , C. M. G.; Jorge, R. M. M.; Coutinho, M. R.; Paraíso, P. R.; Jorge, L. M. de M. Modelagem matemática e análise da hidratação de grãos de ervilha. Ciência e Tecnologia de Alimentos, v.29, p.12-18, 2009. http://dx.doi.org/10.1590/ S0101-20612009000100003

Peleg, M. An empirical model for the description of moisture sorption curves. Journal of Food Science, v.52, p.1216-1219, 1988. http:// dx.doi.org/10.1111/j.1365-2621.1988.tb13565.x

Reitz, P. R. Flora Ilustrada catarinense. Itajai: Herbário Barbosa Rodrigues e Editora Atlas SA, 1982.321p.

Resende, O.; Corrêa, P. C. Modelagem matemática do processo de hidratação de sementes de feijão, Acta Scientiarum Agronomy, v.29, p.373-378, 2007. http://dx.doi.org/10.4025/actasciagron. v29i3.387

Resende, O.; Ferreira, L. U.; Almeida, D. P. Modelagem matemática para descrição da cinética de secagem do feijão Adzuki. Revista Brasileira de Produtos Agroindustriais, v.12, p.171178, 2010.

Resende, O.; Rodrigues, E.; Mendes, U. C.; Almeida, D. P. Hidratação e difusividade efetiva do feijão adzuki (Vigna angularis). Revista Brasileira de Armazenamento, v.34, p.122-128, 2009.

Roca, E.; Guillard, V.; Broyart, B.; Guilbert, S.; Gontard, N. Effective moisture diffusivity modeling food structure and hygroscopicity. Food Chemistry, v.106, p.1428-1437, 2008. http://dx.doi. org/10.1016/j.foodchem.2007.03.078

Schwaab, M.; Pinto, J. C. Análises de dados experimentais I Fundamentos de estatística e estimação de parâmetros. 1.ed. Rio de Janeiro: E-papers, 2007. 125p.

Senadeera, W.; Bhandari, B. R.; Young, G.; Wijesinghe, B. Influence of shapes of selected vegetable materials on drying kinetics during fluidized bed drying. Journal of Food Engineering, v.58, p.277-283, 2003. http://dx.doi.org/10.1016/S02608774(02)00386-2

Siqueira, V. C.; Resende, O.; Chaves, T. H. Difusividade efetivo de grãos e frutos de pinhão-manso. Semina: Ciências Agrárias, v.33, p.2919-2930, 2012.

Turhan, M.; Sayar, S.; Gunasekaran, S. Application of Peleg model to study water absorption in chickpea during soaking. Journal of Food Engineering, v.53, p.153-159, 2002. http://dx.doi. org/10.1016/S0260-8774(01)00152-2

Woodstock, L. W. Seed imbibition: A critical period for successful germination. Journal of Seed Technology, v.12, p.1-15, 1988. 